\title{
Antibacterial Effects of Capparis decidua Fruit Mediated Selenium Nanoparticles
}

\author{
Shabbarish Chockalingam ${ }^{1}$, Preetha $\mathrm{S}^{2}$, Jeevitha $\mathrm{M}^{3}$, Lavanya Pratap ${ }^{4}$ \\ ${ }^{1}$ Department of Physiology, Saveetha Dental College and Hospitals, Saveetha Institute of Medical and Technical \\ Sciences (SIMATS), Chennai, Tamil Nadu, India. ${ }^{2}$ Department of Physiology, Saveetha Dental College and \\ Hospitals, Saveetha Institute of Medical and Technical Sciences (SIMATS), Chennai, Tamil Nadu, India. \\ ${ }^{3}$ Department of Periodontics, Saveetha Dental College and Hospitals, Saveetha Institute of Medical and Technical \\ Sciences (SIMATS), Chennai, Tamil Nadu, India. ${ }^{4}$ Department of Anatomy, Saveetha Dental College and \\ Hospitals, Saveetha Institute of Medical and Technical Sciences (SIMATS), Chennai, Tamil Nadu, India.
}

\section{ABSTRACT}

\section{BACKGROUND}

Capparis decidua and Selenium have several antibacterial properties along with other highly desirable properties, but for long they have not been explored. Nanoparticles are efficient in transporting drugs as they are easily absorbed by the body due to their small size. We wanted to evaluate the antibacterial activity of Capparis decidua mediated selenium nanoparticles against Streptococcus mutans, Lactobacillus, and Enterococcus faecalis.

\section{METHODS}

4 wells were dug in three Petri dishes of Agar which were coated with a layer of bacteria, Streptococcus mutans, Lactobacillus and Enterococcus faecalis. The wells were filled with different amounts of the extract in the order of $50 \mu \mathrm{L}, 100 \mu \mathrm{L}$ and 150 $\mu \mathrm{L}$ with the fourth well having $50 \mu \mathrm{L}$ of the antibiotic. Zone of inhibition was measured.

\section{RESULTS}

After the measurements were made, the results obtained showed good zone of inhibition against Streptococcus mutans, Lactobacillus and Enterococcus faecalis.

\section{CONCLUSIONS}

This study suggests that Capparis decidua fruit mediated with Selenium Nanoparticles have a high antibacterial activity.

\section{KEY WORDS}

Antibacterial Effects, Capparis decidua, Selenium, Nanoparticles
Corresponding Author:

Dr. Preethas.

Department of Physiology,

Saveetha Dental College and Hospitals,

Saveetha Institute of Medical and

Technical Sciences (SIMATS),

Saveetha University162,

Poonamallee High Road

Chennai- 600 077, Tamil Nadu, India

E-mail: drpreeth.homeo@gmail.com

DOI: $10.14260 / j e m d s / 2020 / 646$

How to Cite This Article:

Chockalingam S, Preetha $S$, Jeevitha $M$, et al Antibacterial effects of Capparis decidua fruit mediated selenium nanoparticles. J Evolution Med Dent Sci 2020;9(40):29472950, DOI: $10.14260 /$ jemds/2020/646

Submission 27-06-2020,

Peer Review 24-08-2020,

Acceptance 31-08-2020,

Published 05-10-2020.

Copyright (C) 2020 Shabbarish Chockalingam et al. This is an open access article distributed under Creative Commons Attribution License [Attribution 4.0 International (CC BY 4.0)] 


\section{BACKGROUND}

Nanoparticles are particles who have a size of $100 \mathrm{~nm}$ or less. They have a high surface area due to their small size. ${ }^{1}$ Nanoparticles have a characteristic array of colours which includes wine red, yellowish grey and black among other colours. ${ }^{2}$ When nanoparticles are plant mediated, they are highly eco-friendly. ${ }^{3}$ This study was conducted in order to know the antibacterial effects of Capparis decidua fruit mediated with Selenium nanoparticles because of the known although highly unexplored potential of the antibacterial properties of Selenium and Capparis decidua. Their small size makes them easily absorbable by the body, thus they serve to be potent carriers of medicine and their larger surface area means delivery of a larger amount of medicine to the body.

Selenium (atomic number 34, atomic mass $78.96 \mathrm{u}$ ) is among the several trace elements present in the body. ${ }^{4}$ It is categorized under essential trace elements as it is not constantly found in living matter. ${ }^{5}$ It is found to have several benefits such as its antibacterial \& broad-spectrum antifungal properties as well as shown effectiveness against malignancies and reducing cancer. ${ }^{6,7,8}$ Nanoparticles of Selenium is better than Sodium Selenite due to lower acute history and it also showed less oxidative effects than Selenite, add to that it's antifungal, antibacterial and anticancer properties makes it the more viable option. ${ }^{8,9}$ Another property of Selenium nanoparticles which make them highly viable is that they found to be far much more biocompatible and non-toxic when compared to Selenite and Selenate. ${ }^{10}$

The antibacterial properties of Selenium nanoparticles were seen against Staphylococcus aureus as they strongly inhibited the growth of the bacteria. It plays an active role in the production of thyroid hormone.

Selenium nanoparticles when synthesized were found to be spherical in shape with a size range of $30 \mathrm{~nm}$ to $150 \mathrm{~nm} .^{11}$ Another highly interesting fact is that Selenium seemed to have had the strongest effect on those individuals who had the lowest Selenium levels. ${ }^{12}$ Selenium facilitates wound healing, in which case we have to apply a Selenium MAP cooked gauze to the tissues of burn wound. ${ }^{13}$ This of course points to the importance of Selenium in our body but in no way suggests that we need to take supplements for this. Nanoparticles of Selenium were first synthesized by Pseudomonas alcaliphila in a method that was both simple as well eco-friendly and its synthesis has always been. ${ }^{14}$ Selenium nanoparticles have been synthesized from a variety of plants including Vitus vinifera, Capsicum annum and Withania somnifera.

Capparis decidua has varying properties which include antifungal, antimicrobial but more importantly antibacterial although these properties can be observed only in different solvents. ${ }^{15}, 16$ the antibacterial properties have not been explored in as much depth as the other properties of this fruit. It is a known fact that broad spectrum antibiotics have unfavourable effects on the human body; this also results in cross reactivity in bodily fluids. ${ }^{17}$ All this has increased the demands for alternatives. Although it has been known to have been effective against Pseudomonas aeruginosa, E. coli and Bacillus subtilis. Factoring in all the points which was brought up above it became evident that as time passes the present antibiotics are going to have lesser and lesser effects gradually over time and these calls for new antibiotics. Enterococcus faecalis causes up to $90 \%$ of all enterococcal diseases,
Lactobacillus has been involved in some clinical conditions that include bacteraemia and infective endocarditis and Streptococcus mutans is a known pathogen of dental caries. ${ }^{18,}$ 19 , and 20

The present study aims to evaluate the antibacterial activity of Capparis decidua mediated selenium nanoparticles against Streptococcus mutans, Lactobacillus, Enterococcus faecalis.

\section{METHODS}

\section{Preparation of Extract}

$1 \mathrm{~g}$ of Capparis decidua fruit powder was mixed in $100 \mathrm{~mL}$ water and was kept in a water bath for 1 hour with the temperature maintained at $90^{\circ} \mathrm{C}$. The extract was then filtered by using Whatman No. 1 filter paper and the prepared extract was kept in a separate beaker for further use. Later, $30 \mathrm{~mL}$ of the prepared extract was further mixed with $70 \mathrm{~mL}$ of distilled water and the prepared solution was used to prepare $30 \mathrm{~mL}$ of sodium selenite. This was kept on a magnetic stirrer for the synthesis of nanoparticles (Figure 1).

\section{Synthesis of Selenium Nanoparticles}

The synthesis of nanoparticles was indicated by the change in colour of the solution and a UV spectroscopy reading was taken at correct intervals. The synthesised nanoparticles were purified by centrifugation and its antibacterial activity against Streptococcus mutans, Lactobacillus and Enterococcus faecalis.

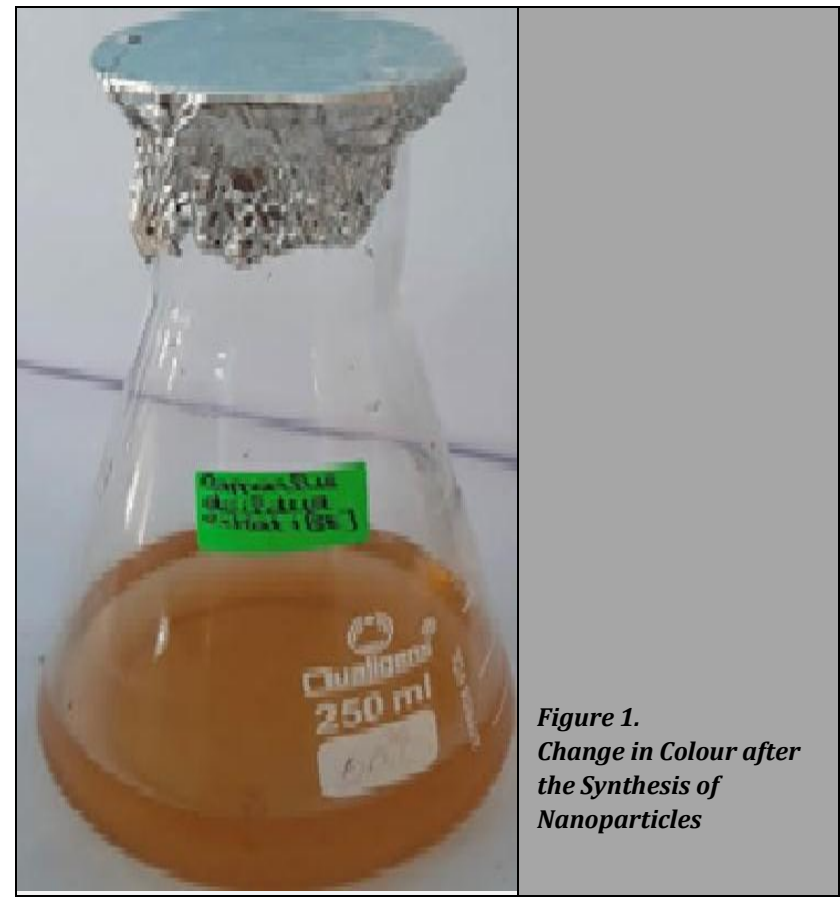

\section{Antibacterial Activity of Synthesized Particles}

The antibacterial activity of the synthesized selenium nanoparticles was determined by using agar well diffusion assay method which is also known as agar disc diffusion method. The sensitivity of the bacteria to the extract is determined by the formation of zone of inhibition around the 
well. Stock cultures of Streptococcus mutans, Lactobacillus and Enterococcus faecalis were prepared and maintained in saturated dextrose agar slants at $4{ }^{\circ} \mathrm{C}$. The petri dish was incubated under suitable conditions and a positive control drug was also done parallel for the comparison of the results. Generally, the antibacterial agents diffuse into the agar and then inhibits the growth of the microorganism, forming a zone around them. The dishes were examined for the presence of a zone of inhibition, characterised by the appearance of a clear area around the wells, the diameters of which were measured using a rule and they were noted down.
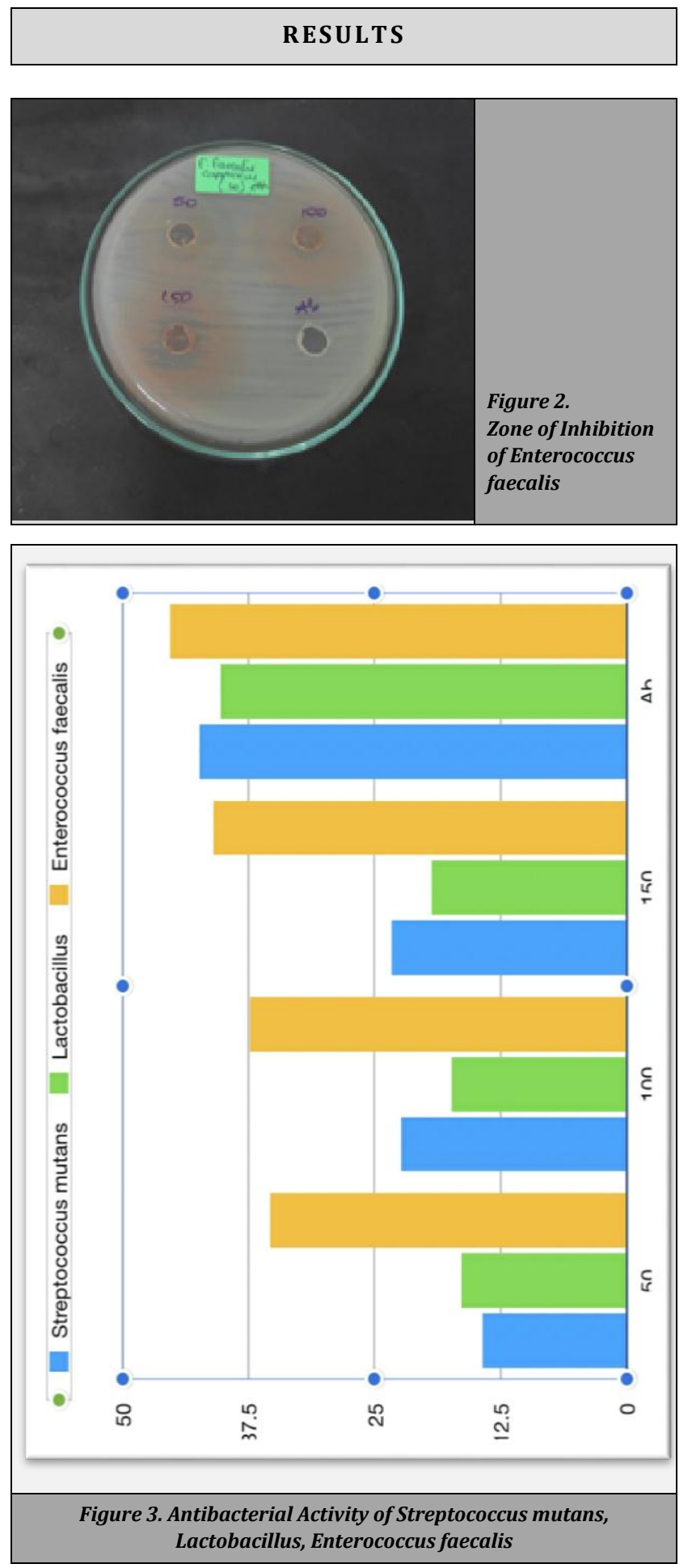

Antibacterial bacterial activity was studied by measuring zone of inhibition in the petri dish by diffusion method. Figure 4 gives the comparison of the effect on all of the samples. For Streptococcus mutans, the diameter of the zone of inhibition for $50 \mu \mathrm{L}$ is $14.33 \mathrm{~mm} \pm 0.47 \mathrm{~mm}$, for $100 \mu \mathrm{L}$ it is $22.33 \mathrm{~mm} \pm$ $0.47 \mathrm{~mm}$ and for $150 \mu \mathrm{L}$ is $23.33 \mathrm{~mm} \pm 0.47 \mathrm{~mm}$. For the positive control drug, it was $42.33 \mathrm{~mm} \pm 0.47 \mathrm{~mm}$. Lactobacillus had a diameter of $16.33 \mathrm{~mm} \pm 0.47 \mathrm{~mm}$ for 50 $\mu \mathrm{L}, 17.33 \mathrm{~mm} \pm 0.47 \mathrm{~mm}$ for $100 \mu \mathrm{L}$ and $19.33 \mathrm{~mm} \pm 0.47 \mathrm{~mm}$ for $150 \mu \mathrm{L}$. the positive control drug had a reading of $40.33 \mathrm{~mm}$ $\pm 0.47 \mathrm{~mm}$. (Table 2) (Figure 2). Enterococcus faecalis upon being subjected to $50 \mu \mathrm{L}$ had a diameter of $35.55 \mathrm{~mm} \pm 0.47$ $\mathrm{mm}, 100 \mu \mathrm{L}$ has $37.33 \mathrm{~mm} \pm 0.47 \mathrm{~mm}$ and $150 \mu \mathrm{L}$ had 40.33 $\mathrm{mm} \pm 0.47 \mathrm{~mm}$. The positive control drug gave a reading of $45.33 \mathrm{~mm} \pm 0.47 \mathrm{~mm}$.

\begin{tabular}{|cccc|}
\hline $\begin{array}{c}\text { Concentration } \\
(\boldsymbol{\mu L})\end{array}$ & $\begin{array}{c}\text { Diameter of Zone of Inhibition (mm) } \\
\text { Streptococcus } \\
\text { mutans }\end{array}$ & Lactobacillus & $\begin{array}{c}\text { Enterococcus } \\
\text { faecalis }\end{array}$ \\
50 & $14.33 \pm 0.47$ & $16.33 \pm 0.47$ & $35.33 \pm 0.47$ \\
100 & $22.33 \pm 0.47$ & $17.33 \pm 0.47$ & $37.33 \pm 0.47$ \\
150 & $23.33 \pm 0.47$ & $19.33 \pm 0.47$ & $40.33 \pm 0.47$ \\
$\mathrm{Ab}$ & $42.33 \pm 0.47$ & $40.33 \pm 0.47$ & $45.33 \pm 0.47$ \\
\hline \multicolumn{4}{|c}{ Table 1. Antibacterial Activity of Capparis decidua } \\
\hline
\end{tabular}

\section{DISCUSSION}

This study was conducted in order to know the antibacterial effects of Capparis decidua fruit mediated with Selenium nanoparticles. This study was taken up because of the known although highly unexplored potential of the antibacterial properties of Selenium and Capparis decidua. From this study, it was found that Capparis decidua had a largely positive effect on the bacteria, used in this study. It managed to do both inhibit and prevent the growth of a large variety of bacteria.

By conducting this study, it confirms the findings of Phang A. Tran, ${ }^{7}$ which suggested a potential antibacterial effect of Selenium nanoparticles. This study only studied Selenium nanoparticles and had nothing more and used a single bacterium as the sample. It leaves a lot of doubt as to its effectiveness against other types of bacteria and does not provide a lot of information on this matter.

Ayat A A Abdalrahman ${ }^{15}$ conducted several researches about the various properties about Capparis decidua as to know about the various fields where it can be used. Sandeep Kumar Dixit ${ }^{21}$ also found that the medium in which the Capparis decidua is kept also matters as ethanolic medium was found to have more antibacterial properties, but in his study, while he used studied both bacteria and fungi, he does not mediate it with anything thus his study only nothing more than the power of Capparis decidua all by itself.

My study while it was focussed Capparis decidua, the effects were compounded with those of Selenium making it a more viable option than the two of these alone. The study by Phang A. Tran ${ }^{7}$ is the only other study that explored the combination of both of these two, yet the possibility of examining the two together on a larger was not explored. This study shows us that this combination of Capparis decidua and Selenium nanoparticles is effective on both Gram-positive bacteria unlike the findings of Soumya Menon which said that it is effective against only Gram-negative bacteria. 
Study by Xiuyong Chen et al $^{22}$ suggests that Selenium is capable of holding up its antibacterial properties for extended periods of time, along with its other properties. It is been found to have the ability of transferring its antibacterial activities to hand towels which gives it a more viable option commercially. ${ }^{23}$ Selenium is also viable against food borne pathogens such as E. coli, Salmonella and Listeria monocytogens. ${ }^{24}$ Capparis decidua, has high antidiabetic values and can be used for the treatment of patients suffering with diabetes while also suffering from an infection. ${ }^{25}$ When delivered with nanoparticles, their size which ranges up to a maximum of $100 \mathrm{~nm}^{26}$ means that they are more effective when it comes to expressing their properties.

The variety of properties that these two are known for mean that there not several studies which study just one property hence there are not many studies which goes into depth about the antibacterial property of Capparis decidua mediated with Selenium nanoparticles.

\section{CONCLUSIONS}

This study shows that, Capparis decidua mediated Selenium nanoparticles have good antibacterial property.

Financial or Other Competing Interests: None.

\section{REFERENCES}

[1] Khan I, Saeed K, Khan I. Nanoparticles: properties, applications and toxicities. Arabian Journal of Chemistry 2019;12(7):908-31.

[2] Horikoshi S, Serpone N. Introduction to Nanoparticles. Microwaves in nanoparticle synthesis: fundamentals and applications. Wiley-VCH Verlag GmbH \& Co. KGaA 2013:1-24.

[3] Keziah VS, Rajeskumar S, Lakshmi T, et al, Free radical scavenging activity of plant-mediated zinc oxide nanoparticles. Drug Invention Today 2019;11(9):2165-8.

[4] Versieck J, McCall JT. Trace elements in human body fluids and tissues. CRC Critical Reviews in Clinical Laboratory Services 1985;22(2):97-184.

[5] Joshi SM, De Britto M, Jogaiah S, et al. Mycogenic selenium nanoparticles as potential new generation broad spectrum antifungal molecules. Biomolecules 2019;9(9)419.

[6] Huang Y, He L, Liu W, et al. Selective cellular uptake and induction of apoptosis of cancer targeted Selenium nanoparticles. Biomaterials 2019;34(29):7106-16.

[7] Tran PA, Webster TJ. Selenium nanoparticles inhibit staphylococcus aureus growth. Int $\mathrm{J}$ of Nanomed 2011;6:1553-8.

[8] Zhang JS, Gao XY, Zhang LD, et al. Biological effects of nano red elemental selenium. Biofactors 2001;15(1):27-38.

[9] Menon S, Devi KSS, Santhiya R, et al. Selenium nanoparticles: a potent chemotherapeutic agent and an elucidation of its mechanism. Colloids Surf B Biointerfaces 2018;170:280-92.

[10] Wadhwani SA, Shedbalkar UU, Singh R, et al. Biogenic selenium nanoparticles prospects. Appl Microbiol Biotech 2016;100(6):2555-66.
[11] Srivastava N, Mukhopadhyay M. Biosynthesis and structural characterization of selenium nanoparticles mediated by Zooglea ramigera. Powder Technology 2013;244:26-9.

[12] Rayman MP. Selenium in cancer prevention: a review of the evidence and mechanism of action. Proc Nutr Soc 2005;64(4):527-42.

[13] Tran PL, Hammond AA, Mosley T. Organoselenium coating on cellulose inhibits the formation of biofilms by pseudomonas aeruginosa and staphylococcus aureus. Appl Environ Microbiol 2009;75(11):3586-92.

[14] Zhang W, Chen Z, Liu H, et al. Biosynthesis and structural characteristics of selenium nanoparticles by pseudomonas alcaliphila. Colloids Surf B Biointerfaces 2011;88(1):196-201.

[15] Abdalrahman AAA, El Tigani S, Yagi S. Biological activity of extracts from Capparis decidua L. twigs. J Med Plants Res 2016;10(1):1-7.

[16] Giridharan T, Masi C, Sindhu S, et al. Studies on green synthesis. characterisation and anti proliferation potential of silver nanoparticles using Dodonea viscosa and Capparis decidua. Biosci Biotech Res Asia 2014;11(2):665-73.

[17] Upadhyay RK, Ahmad S, Tripathi R, et al. Screening of antimicrobial potential of extracts and pure compounds isolated from Capparis decidua. J Med Plan Res 2010;4(6):439-45.

[18] Kumar SA, Ajitha P, Prakasam G, et al. Genotypic and phenotypic detection of certain virulent determinates of Enterococcus faecalis associated with dental caries-an in vitro study. IOSR Journal of Dental and Medical Sciences (IOSR-JDMS) 2018;17(12):67-71.

[19] Sherid M, Samo S, Sulaiman S, et al. Liver abscess and bacteremia caused by Lactobacillus: role of probiotics? Case report and review of the literature. BMC Gastroenterol 2016;16(1):138.

[20] Nakano K, Nomura R, Ooshima T. Streptococcus mutans and cardiovascular diseases. Japanese Dental Science Review 2008;44(1):29-37.

[21] Dixit SK, Kumari P, Prajapati R, et al. Characterization \& evaluation of antibacterial, antifungal activity of environment friendly Capparis decidua microemulsion. International Journal of Pharmacy and Pharmaceutical Sciences 2014;17(1):255-7.

[22] Chen X, Cai K, Fang J, et al. Fabrication of seleniumdeposited and chitosan-coated titania nanotubes with anticancer and antibacterial properties. Colloids Surf B Biointerfaces 2013;103:149-57.

[23] Nguyen THD, Vardhanabhuti B, Lin M, et al. Antibacterial properties of selenium nanoparticles and their toxicity to Caco-2 cells. Food Control 2017;77:17-24.

[24] Wang Q, Webster TJ. Short communication: inhibiting biofilm formation on paper towels through the use of selenium nanoparticles coatings. Int J Nanomedicine 2012;8:407-11.

[25] Zia-Ul-Haq M, Ćavar S, Qayum M, et al. Compositional studies: antioxidant and antidiabetic activities of Capparis decidua (Forsk.) Edgew. Int J Med Sci 2011;12(12):884661.

[26] Radad K, Al-Shraim M, Moldzio R, et al. Recent advances in benefits and hazards of engineered nanoparticles. Environ Toxicol Pharmacol 2012;34(3):661-72. 